\title{
STABILIZATION OF THE KORTEWEG-DE VRIES EQUATION WITH LOCALIZED DAMPING
}

BY

G. PERLA MENZALA (National Laboratory of Scientific Computation, LNCC/MCT, Rua Getulio Vargas 333, Quitandinha, Petrópolis, RJ, CEP 25651-070, RJ, Brasil and IMUFRJ, P.O. Box 68530, Rio de Janeiro, RJ, Brasil),

C. F. VASCONCELloS (IMUFRJ, P.O. Box 68530, Rio de Janeiro, RJ, Brasil),

AND

E. ZUAZUA (Departamento de Matemática Aplicada, Universidad Complutense de Madrid, 28040 Madrid, Spain)

Abstract. We study the stabilization of solutions of the Korteweg-de Vries (KdV) equation in a bounded interval under the effect of a localized damping mechanism. Using multiplier techniques we deduce the exponential decay in time of the solutions of the underlying linear equation. A locally uniform stabilization result of the solutions of the nonlinear $\mathrm{KdV}$ model is also proved. The proof combines compactness arguments, the smoothing effect of the $\mathrm{KdV}$ equation on the line and unique continuation results.

1. Introduction. We consider the Korteweg-de Vries (KdV) equation in a bounded interval $(0, L)$ under the presence of a localized damping

$$
\left\{\begin{array}{l}
u_{t}+u_{x}+u_{x x x}+u u_{x}+a(x) u=0 \text { in }(0, L) \times(0,+\infty) \\
u(0, t)=u(L, t)=0 \text { for all } t>0 \\
u_{x}(L, t)=0 \text { for all } t>0 \\
u(x, 0)=u_{0}(x) \text { in }(0, L)
\end{array}\right.
$$

Here $a=a(x)$ is a nonnegative function belonging to $L^{\infty}(0, L)$. In most of the paper we will also assume that $a(x) \geq a_{0}>0$ a.e. in an open, nonempty subset $\omega$ of $(0, L)$. Therefore, the damping term is acting effectively in $\omega$.

The KdV equation (1.1), in the absence of damping, models the (unidirectional) propagation of water waves of small amplitude in a bounded channel and has been the object of intensive research (see, for instance, [1], [13], and [24] and the references therein).

\footnotetext{
Received December 20, 1999.

2000 Mathematics Subject Classification. Primary 35B40, 35Q53.

E-mail address: perla@lncc.br

E-mail address: fred@dmm.im.ufrj.br

E-mail address: zuazua@eucmax.sim.ucm.es
} 
The total energy associated with (1.1) is given by

$$
E(t)=\frac{1}{2} \int_{0}^{L}|u(x, t)|^{2} d x
$$

Under the above boundary conditions it is easy to prove that

$$
\frac{d E}{d t}=-\int_{0}^{L} a(x)|u(x, t)|^{2} d x-\frac{1}{2}\left|u_{x}(0, t)\right|^{2} \leq 0
$$

for all $t>0$. Consequently, $E(t)$ is a nonincreasing function. The following basic questions arise: Does $E(t) \rightarrow 0$ as $t \rightarrow+\infty$ and, if yes, is it possible to find a rate of decay of $E(t)$ ?

Observe that, according to the above energy dissipation law, even when $a \equiv 0$, the energy $E$ is dissipated through the extreme $x=0$.

Our analysis (inspired by the work by Rosier [15] on the controllability problem) of the linearized problem (around $u=0$ ) shows that, when $a=0$, the decay of solutions fails for some critical values of the length of the interval $L$. Therefore, it is natural to introduce an extra damping term in order to guarantee the decay. Here we are concerned with the case where the dissipation is localized in some strict subset $\omega$ of $[0, L]$.

When $a \equiv 1$ it is straightforward to see from the energy dissipation law that the energy decays uniformly exponentially as $t \rightarrow+\infty$. The same holds when $a(x) \geq a_{0}>0$ a.e. in $[0, L]$. The problem of stabilization when the damping is effective only on a subset of the interval $(0, L)$ is much more subtle.

This problem has been extensively investigated in the context of wave equations and there is a large literature on the subject. For instance, in the articles by Dafermos [3], Haraux [4] and Slemrod [23], La Salle's invariance principle was used as a main tool to obtain asymptotic stability properties. More recently, assuming that the dissipation is effective in a suitable subset of the domain where the equation holds, decay rates have been obtained (see for instance Zuazua [28] and Nakao [14]).

The same problem has also been addressed for the KdV equation. This has been done by means of two different damping mechanisms with periodic boundary conditions. First, we can mention the work [8] where a damping mechanism, distributed all along the domain, that guarantees the conservation of mass was introduced. In [8] it was shown that solutions converge exponentially to the averaged constant solution as time tends to infinity (see also [7]). The same was done by Russell and Zhang in [19] and [20] by means of a damping mechanism with localized support, periodic boundary conditions and small initial data. The same problem has also been addressed by means of a boundary damping on the periodic boundary conditions (see [18]).

There are fewer results for the boundary conditions as in (1.1). However, there are quite complete results in what concerns boundary controllability. Rosier in [15] proved that the underlying linear equation without damping is exactly controllable by means of a single boundary control except when $L$ lies in a countable set of critical lengths. This was done using multiplier techniques and Lions' HUM method (see [10]). The 
critical lengths are such that there are eigenfunctions of the linear problem for which the observability inequality leading to controllability fails. In the context of the stabilization problem, they correspond to solutions that, when $a=0$, are undamped, i.e., such that $u_{x}(0, t)=0$ for all time $t$. By a linearization argument a local controllability result for the semilinear equation was also proved in [15]. Later on, Zhang in [27] proved that using three controls, acting on all the boundary conditions, controllability holds for all values of $L$.

Here we are strongly inspired by the work of Rosier [15]. First of all, we analyze the linearized equation (around zero). We observe that, due to the inequalities obtained in [15], the exponential decay of the solutions holds without adding the internal damping term $a(\cdot) u$ provided $L$ does not belong to the set of critical lengths mentioned above. When $L$ is one of these critical lengths, the exponential decay fails but only because of the existence of a finite-dimensional space of solutions that are completely undamped. This suggests that, adding an extra damping (like the one $a(\cdot) u$ provides, for instance), the exponential decay of the solutions of the linear equation will hold for all values of the length $L$. This is proved to be true.

We then analyze the nonlinear $\mathrm{KdV}$ equation. First of all, for the sake of completeness, we give a sketch of the proof of the existence and uniqueness of global solutions. Then, we show that, as a consequence of the exponential decay of the underlying linear semigroup, small amplitude solutions of the nonlinear problem decay exponentially as well. The main contribution of this paper is to show that large solutions decay exponentially as well when the damping is active simultaneously in a neighborhood of both extremes $x=0, L$. Our result is of local nature in the sense that the exponential decay rate is uniform on bounded sets of initial data. We argue, as in the linear case, using multipliers. However, the nonlinearity produces extra terms that we handle by compactness. The problem is then reduced to showing that the unique solution of $(1.1)$ such that $a(\cdot) u=0$ everywhere and $u_{x}(0, t) \equiv 0$ for all time is the trivial one. This requires the application of a unique continuation result as in [26]. However, this unique continuation result may not be applied directly because of the lack of regularity of the solutions we are dealing with. Therefore, we have first to show that these solutions are smooth enough. Taking into account that $a(\cdot) u=0$ and that $a>0$ in a neighborhood of both extremes $x=0$, we deduce that $u$ has compact support in $x \in(0, L)$. This allows us to extend the solution by zero outside the interval $(0, L)$ to obtain a solution of the KdV equation on the whole line. We can then apply the classical smoothing properties of this equation as in [6] and $[9]$.

The problem is open in the case where $a>0$ in an open subset of $(0, L)$ that does not contain a neighborhood of both extremes. The reason for that is that we do not have enough regularity of the weak solution satisfying $a(\cdot) u=0$ to apply the existing unique continuation results. This is probably a purely technical problem that could be overcome by proving unique continuation results for weaker solutions. But, as far as we know, this remains to be done.

The article is organized as follows. In Section 2 we analyze the linearized problem around the zero solution. Section 3 is devoted to the semilinear problem. In Section 4 we briefly discuss some open problems on unique continuation mentioned above and possible extensions. 
2. The linearized $\mathbf{K d V}$ equation. In this section we are interested in the exponential stability of the solutions $u=u(x, t)$ of the following problem:

$$
\left\{\begin{array}{l}
u_{t}+u_{x}+u_{x x x}+a(x) u=0 \text { in } I \times \mathbb{R}^{+} \\
u(0, t)=u(L, t)=0 \text { for all } t>0 \\
u_{x}(L, t)=0 \text { for all } t>0 \\
u(x, 0)=u_{0}(x) \text { for all } x \in I
\end{array}\right.
$$

where $I=\{0<x<L\}$ and $a \in L^{\infty}(I), a \geq 0$ and $a(x) \geq a_{0}>0$ a.e. in an open, nonempty subset $\omega$ of $I$.

Obviously, (2.1) is the linearization of (1.1) around the trivial solution $u=0$.

The initial data $u_{0}$ is assumed to be in $L^{2}(I)$. First, we consider the case $a \equiv 0$.

2.1. The case $a \equiv 0$. The well-posedness of problem (2.1) with $a \equiv 0$ was proved by Rosier [15] showing that the operator $A=-\frac{\partial^{3}}{\partial x^{3}}-\frac{\partial}{\partial x}$ with domain $D(A)=\{w \in$ $\left.H^{3}(I), w(0)=w(L)=w_{x}(L)=0\right\} \subseteq L^{2}(I)$ is the infinitesimal generator of a strongly continuous semigroup of contractions in $L^{2}(I)$.

When analyzing the controllability of system (2.1) with $a \equiv 0$, Rosier in [15] introduced the following exceptional set for the length $L$ of the interval $I$ :

$$
\mathcal{E}=\left\{\frac{2 \pi}{\sqrt{3}} \sqrt{k^{2}+k l+l^{2}}, k \text { and } l \text { are positive natural numbers }\right\} .
$$

Rosier proved in Lemma 3.5 of [15] that if $L$ belongs to $\mathcal{E}$, then there exist complex numbers $\lambda$ and $u_{0} \in H^{3}(I), u_{0} \not \equiv 0$ such that

$$
\begin{aligned}
& \lambda u_{0}+\frac{d u_{0}}{d x}+\frac{d^{3} u_{0}}{d x^{3}}=0 \quad \text { in } 0<x<L, \\
& u_{0}(0)=\frac{d u_{0}(0)}{d x}=u_{0}(L)=\frac{d u_{0}(L)}{d x}=0 .
\end{aligned}
$$

Observe that, whenever $u_{0}$ solves $(2.3), u(x, t)=u_{0}(x) e^{\lambda t}$ is a solution of (2.1) with $a \equiv 0$ and satisfies

$$
\frac{d}{d t} \int_{0}^{L}|u(x, t)|^{2} d x=0
$$

because

$$
u_{x}(0, t)=\frac{d u_{0}(0)}{d x} e^{\lambda t}=0
$$

This shows that when $L$ belongs to $\mathcal{E}$ there are solutions of $(2.1)$ with $a \equiv 0$ that do not decay.

It is also important to note that, as proved in [15], when $L \in \mathcal{E}$, the subspace of undamped solutions is finite-dimensional.

The following result provides an exponential decay rate when $L$ does not belong to $\mathcal{E}$. 
ThEOREM 2.1. Consider the solution of the following problem:

$$
\left\{\begin{array}{l}
u_{t}+u_{x}+u_{x x x}=0 \text { in } I \times \mathbb{R}^{+} \\
u(0, t)=u(L, t)=0 \text { for all } t>0 \\
u_{x}(L, t)=0 \text { for all } t>0 \\
u(x, 0)=u_{0}(x) \text { for all } x \in I
\end{array}\right.
$$

If $L$ does not belong to $\mathcal{E}$, then there exist $c>0$ and $\mu>0$ such that

$$
\|u(\cdot, t)\|_{L^{2}(I)}^{2} \leq c\left\|u_{0}\right\|_{L^{2}(I)}^{2} e^{-\mu t}
$$

for all $t \geq 0$ and $u_{0} \in L^{2}(I)$.

Proof. In this proof we follow closely the multiplier techniques developed in [15] in the context of the controllability problem.

Let us multiply equation $(2.4)$ by $u$ and integrate in $I=(0, L)$ to obtain

$$
\frac{1}{2} \frac{d}{d t}\|u(\cdot, t)\|_{L^{2}(I)}^{2}+\frac{1}{2} \int_{0}^{L}\left(u^{2}\right)_{x} d x+\int_{0}^{L} u u_{x x x} d x=0 .
$$

Since

$$
\int_{0}^{L}\left(u^{2}\right)_{x} d x=0 \text { and } \int_{0}^{L} u u_{x x x} d x=-\int_{0}^{L} u_{x} u_{x x} d x
$$

we obtain

$$
\frac{1}{2} \frac{d}{d t}\|u(\cdot, t)\|_{L^{2}(I)}^{2}=\frac{1}{2} \int_{0}^{L}\left(u_{x}^{2}\right)_{x} d x
$$

or, equivalently,

$$
\frac{d}{d t}\|u(\cdot, t)\|_{L^{2}(I)}^{2}=-u_{x}^{2}(0, t) \leq 0 .
$$

Next, we use Proposition 3.3 in [15], which guarantees that if $L \notin \mathcal{E}$, given $T>0$ there exists $c>0(c=c(L, T))$ such that

$$
\left\|u_{0}\right\|_{L^{2}(I)}^{2} \leq c\left\|u_{x}(0, \cdot)\right\|_{L^{2}(0, T)}^{2}
$$

for every solution of (2.4). Integration of identity (2.5) on $(0, T)$ and using (2.6) give us

$$
\begin{aligned}
(1+c)\|u(\cdot, T)\|_{L^{2}(I)}^{2}= & \left\|u_{0}\right\|_{L^{2}(I)}^{2}-c \int_{0}^{T} u_{x}^{2}(0, s) d s \\
& +c\left\|u_{0}\right\|_{L^{2}(I)}^{2}-\int_{0}^{T} u_{x}^{2}(0, s) d s \\
\leq & c\left\|u_{0}\right\|_{L^{2}(I)}^{2}-\int_{0}^{T} u_{x}^{2}(0, s) d s \\
\leq & c\left\|u_{0}\right\|_{L^{2}(I)}^{2},
\end{aligned}
$$

which implies that

$$
\|u(\cdot, T)\|_{L^{2}(I)}^{2} \leq\left(\frac{c}{c+1}\right)\left\|u_{0}\right\|_{L^{2}(I)}^{2},
$$

that is, $E(T) \leq \gamma E(0)$ with $0<\gamma<1$. The semigroup property implies the conclusion of Theorem 2.1 . 
Theorem 2.1 is sharp since, as indicated above, when the length $L$ belongs to the set $\mathcal{E}$ of critical lengths, there are nontrivial solutions that are undamped and that, consequently, do not decay.

2.2. The case $a \not \equiv 0$. In what concerns the well-posedness of system (2.1), the case when $a \not \equiv 0$ can be handled in a similar way by considering (2.1) as a perturbation of the case $a \equiv 0$.

In what concerns stabilization, the following holds.

Theorem 2.2. Assume that $a>0$ in an open nonempty subset $\omega$ of $(0, L)$. Then, for any $L>0$, there exist $c>0$ and $\mu>0$ such that

$$
E(t) \leq c\left\|u_{0}\right\|_{L^{2}(I)}^{2} e^{-\mu t}
$$

for all $t \geq 0$ and every solution of (2.1) with $u_{0} \in L^{2}(I)$.

REMARK 2.1. Theorem 2.2 guarantees the exponential stability of system (2.1) when the additional damping mechanism provided by $a(x)$ is introduced. This is natural to expect since, as we saw before, when $a \equiv 0$ and $L \notin \mathcal{E}$ the system was already stabilized, and when $L \in \mathcal{E}$ the stabilization failed only because of the existence of a finite-dimensional subspace of undamped solutions. Under these circumstances, any other damping mechanism, effective on all solutions, is expected to provide the exponential decay. This turns out to be the case when the damping is effective on an open subset $\omega$ of $I$. We shall return to this question in Remark 2.2 below.

Proof. Multiply equation $(2.1)$ by $u$ and integrate in $I=(0, L)$ to obtain

$$
\frac{1}{2} \frac{d}{d t}\|u(\cdot, t)\|_{L^{2}(I)}^{2}+\frac{1}{2}\left|u_{x}(0, t)\right|^{2}+\int_{0}^{L} a(x)|u|^{2} d x=0 .
$$

We claim that for any $T>0$, there exists $c=c(T)>0$ such that

$$
\left\|u_{0}\right\|_{L^{2}(I)}^{2} \leq c\left[\int_{0}^{T}\left|u_{x}(0, t)\right|^{2} d t+2 \int_{0}^{T} \int_{0}^{L} a(x)|u(x, t)|^{2} d x d t\right] .
$$

In fact, let us multiply equation $(2.1)$ by $x u$ and integrate on $(0, L) \times(0, T)$ to obtain, after integration by parts and using the boundary conditions,

$$
\begin{aligned}
\int_{0}^{T} \int_{0}^{L} x u u_{t} d x d t & =\frac{1}{2} \int_{0}^{L} x u^{2}(x, T) d x-\frac{1}{2} \int_{0}^{L} x u_{0}^{2} d x \\
\int_{0}^{T} \int_{0}^{L} x u u_{x} d x d t & =-\frac{1}{2} \int_{0}^{T} \int_{0}^{L} u^{2} d x d t \\
\int_{0}^{T} \int_{0}^{L} x u u_{x x x} d x d t & =\frac{3}{2} \int_{0}^{T} \int_{0}^{L} u_{x}^{2} d x d t
\end{aligned}
$$

and

$$
\int_{0}^{T} \int_{0}^{L} x u a(x) u d x d t=\int_{0}^{T} \int_{0}^{L} x a(x) u^{2} d x d t
$$


Adding the above identities we obtain

$$
\begin{aligned}
\int_{0}^{T} \int_{0}^{L} u_{x}^{2} d x d t & +\frac{1}{3} \int_{0}^{L} x u^{2}(x, T) d x+\frac{2}{3} \int_{0}^{T} \int_{0}^{L} x a(x) u^{2} d x d t \\
& =\frac{1}{3} \int_{0}^{T} \int_{0}^{L} u^{2} d x d t+\frac{1}{3} \int_{0}^{L} x u_{0}^{2} d x .
\end{aligned}
$$

Since $a(x) \geq 0$ it follows from (2.13) and (2.7) that

$$
\|u\|_{L^{2}\left(0, T ; H_{0}^{1}(I)\right)}^{2} \leq \frac{3 T+L}{3}\left\|u_{0}\right\|_{L^{2}(I)}^{2} .
$$

Next, we multiply equation $(2.1)$ by $(T-t) u$ and integrate on $(0, L) \times(0, T)$ to obtain the identity

$$
\begin{aligned}
T \int_{0}^{L} u_{0}^{2} d x & =\int_{0}^{T} \int_{0}^{L}|u|^{2} d x d t+\int_{0}^{T}(T-t)\left|u_{x}(0, t)\right|^{2} d t \\
& +2 \int_{0}^{T} \int_{0}^{L}(T-t) a(x)|u|^{2} d x d t .
\end{aligned}
$$

Consequently,

$$
\begin{aligned}
\left\|u_{0}\right\|_{L^{2}(I)}^{2} \leq & \frac{1}{T} \int_{0}^{T} \int_{0}^{L}|u|^{2} d x d t+\int_{0}^{T}\left|u_{x}(0, t)\right|^{2} d t \\
& +2 \int_{0}^{T} \int_{0}^{L} a(x)|u|^{2} d x d t .
\end{aligned}
$$

Therefore, in order to show (2.8) it suffices to prove

$$
\int_{0}^{T} \int_{0}^{L}|u|^{2} d x d t \leq c_{1}\left\{\int_{0}^{T}\left|u_{x}(0, t)\right|^{2} d t+2 \int_{0}^{T} \int_{0}^{L} a(x)|u|^{2} d x d t\right\}
$$

for some positive constant $c_{1}$, independent of the solution $u$.

Let us argue by contradiction following the so-called "compactness-uniqueness" argument (see [28], [29]). Suppose that (2.16) is not valid. Then, there will exist a sequence of solutions $u_{n}$ of $(2.1)$ such that

$$
\lim _{n \rightarrow+\infty} \frac{\left\|u_{n}\right\|_{L^{2}\left(0, T ; L^{2}(I)\right)}^{2}}{\int_{0}^{T}\left|\frac{\partial}{\partial x} u_{n}(0, t)\right|^{2} d t+2 \int_{0}^{T} \int_{0}^{L} a(x)\left|u_{n}\right|^{2} d x d t}=+\infty .
$$

Let $\lambda_{n}=\left\|u_{n}\right\|_{L^{2}\left(0, T ; L^{2}(I)\right)}$ and $v_{n}(x, t)=u_{n}(x, t) / \lambda_{n}$. Clearly, $v_{n}$ solves the problem (2.1) with initial data $u_{n}(x, 0) / \lambda_{n}$. Furthermore,

$$
\left\|v_{n}\right\|_{L^{2}\left(0, T ; L^{2}(I)\right)}=1
$$

and

$$
\int_{0}^{T}\left|\frac{\partial}{\partial x} v_{n}(0, t)\right|^{2} d t+2 \int_{0}^{T} \int_{0}^{L} a(x)\left|v_{n}\right|^{2} d x d t \rightarrow 0
$$

as $n \rightarrow+\infty$.

In view of $(2.15)$ it follows that $v_{n}(x, 0)$ is bounded in $L^{2}(I)$ with $I=(0, L)$. Thus

$$
\left\|v_{n}(\cdot, t)\right\|_{L^{2}(I)} \leq M \quad \text { for all } 0 \leq t \leq T \text {. }
$$


According to (2.14),

$$
\left\|v_{n}\right\|_{L^{2}\left(0, T ; H_{0}^{1}(I)\right)}^{2} \leq\left(\frac{4 T+L}{3}\right)\left\|v_{n}(\cdot, 0)\right\|_{L^{2}(I)}^{2} \leq\left(\frac{4 T+L}{3}\right) M^{2}
$$

for all $n \in \mathbb{N}$. Estimate (2.20) together with (2.18) tells us that

$$
\left(v_{n}\right)_{t}=-\left(v_{n}\right)_{x}-\left(v_{n}\right)_{x x x}-a(x) v_{n}
$$

is bounded in $L^{2}\left(0, T ; H^{-2}(I)\right)$. Since the embedding $H_{0}^{1}(I) \hookrightarrow L^{2}(I)$ is compact, it follows by using classical compactness results (see [22]) that $\left\{v_{n}\right\}$ is relatively compact in $L^{2}\left(0, T ; L^{2}(I)\right)$. By extracting subsequences we may deduce that

$$
v_{n} \rightarrow v \quad \text { weakly in } L^{2}\left(0, T ; H_{0}^{1}(I)\right) \cap H^{1}\left(0, T ; H^{-2}(I)\right)
$$

and

$$
v_{n} \rightarrow v \quad \text { strongly in } L^{2}\left(0, T ; L^{2}(I)\right)
$$

Here to simplify the notation, we denote the subsequence by the index $n$.

Since $\left\|v_{n}\right\|_{L^{2}\left(0, T ; L^{2}(I)\right)}=1$, then

$$
\|v\|_{L^{2}\left(0, T ; L^{2}(I)\right)}=1
$$

By weak lower semicontinuity we have

$$
\begin{aligned}
0 & =\liminf _{k \rightarrow \infty}\left\{\int_{0}^{T}\left|\frac{\partial}{\partial x} v_{n_{k}}(0, t)\right|^{2} d t+2 \int_{0}^{T} \int_{0}^{L} a(x)\left|v_{n_{k}}\right|^{2} d x d t\right\} \\
& \geq \int_{0}^{T}\left|\frac{\partial}{\partial x} v(0, t)\right|^{2} d t+2 \int_{0}^{T} \int_{0}^{L} a(x)|v|^{2} d x d t
\end{aligned}
$$

which guarantees that $a(x) v \equiv 0$ and, in particular, $v \equiv 0$ in $\omega \times(0, T)$. On the other hand, the limit $v$ satisfies

$$
v_{t}+v_{x}+v_{x x x}=0
$$

By Holmgren's Uniqueness Theorem we deduce that $v \equiv 0$ on $I \times(0, T)$. This contradicts (2.21). Consequently (2.16) has to be valid. This shows that (2.8) holds.

On the other hand,

$$
\frac{1}{2} \frac{d}{d t}\|u(\cdot, t)\|_{L^{2}(I)}^{2}+\int_{0}^{L} a(x) u^{2} d x=\frac{1}{2} \int_{0}^{L}\left(u_{x}^{2}\right)_{x} d x=-\frac{1}{2} u_{x}^{2}(0, t) \leq 0
$$

or

$$
\|u(\cdot, T)\|_{L^{2}(I)}^{2}=\left\|u_{0}\right\|_{L^{2}(I)}^{2}-\int_{0}^{T}\left|u_{x}(0, s)\right|^{2} d s-2 \int_{0}^{T} \int_{0}^{L} a(x) u^{2} d x d t
$$


which together with (2.8) gives us the inequality

$$
\begin{aligned}
(1+c)\|u(\cdot, T)\|_{L^{2}(I)}^{2} & =(1+c)\left[\left\|u_{0}\right\|_{L^{2}(I)}^{2}-\int_{0}^{T}\left|u_{x}(0, t)\right|^{2} d t+2 \int_{0}^{T} \int_{0}^{L} a(x)|u|^{2} d x d t\right] \\
& \leq c\left\|u_{0}\right\|_{L^{2}(I)}^{2}-\left[\int_{0}^{T}\left|u_{x}(0, t)\right|^{2} d t+2 \int_{0}^{T} \int_{0}^{L} a(x)|u|^{2} d x d t\right] \\
& \leq c\left\|u_{0}\right\|_{L^{2}(I)}^{2} .
\end{aligned}
$$

Consequently,

$$
\|u(\cdot, T)\|_{L^{2}(I)}^{2} \leq \gamma\left\|u_{0}\right\|_{L^{2}(I)}^{2} \quad \text { with } 0<\gamma=\frac{c}{c+1}<1 .
$$

Therefore, by the semigroup property, the conclusion of Theorem 2.2 follows.

REMARK 2.2. It is interesting to analyze the proof of Theorem 2.2 to understand the amount of damping that is needed to guarantee the exponential decay to hold. Going back to the compactness-uniqueness argument, one sees that the limit solution $v$ is such that:

(a) $v_{x}(0, t) \equiv 0, \quad \forall t>0$

(b) $v \equiv 0$ in $\omega \times(0, T)$.

As indicated above, when $L$ does not belong to $\mathcal{E}$, condition (a) suffices to guarantee that $v \equiv 0$ and this yields the contradiction. When $L \in \mathcal{E}$ the analysis in [15] shows that $v$ lies in a finite-dimensional subspace of solutions of $(2.1)$ such that $v_{x}(0, t) \equiv 0$, which is generated by a finite number of eigenfunctions $\varphi_{1}, \ldots, \varphi_{K}$ satisfying (2.3). In order to guarantee that $v \equiv 0$ we use (b). It is then sufficient to show that the restrictions of $\varphi_{j}$ to the set $\omega=\{a>0\}$ are linearly independent. This has been shown to be true by means of Holmgren's Uniqueness Theorem, but could also be derived directly by analyzing the eigenvalue problem (2.3).

Generally speaking, if we replace the damping mechanism $a(\cdot) u$ by $B u, B$ being a linear bounded operator from $L^{2}(I)$ into itself, such that $\int_{I} B u u d x \geq 0$, exponential decay will hold for all values of the length $L$ if the operator $B$ is such that the $B \varphi_{j}$ 's are linearly independent. But this is a sufficient and not necessary condition. It could also be that the $B \varphi_{j}$ 's are not linearly independent and still the decay holds because of time evolution (since the eigenvalues corresponding to the eigenfunctions $\varphi_{j}$ are all distinct).

Roughly speaking, one can say that, when $L \in \mathcal{E}$, a very weak amount of additional damping stabilizes the system. In particular, we do not need a damping mechanism that dissipates the $L^{2}$-norm as $a(\cdot) u$ does. It would suffice to dissipate some $H^{-s}$-norm. For instance, one could take the damping

$$
B u=1_{\omega}\left(-d^{2} / d x^{2}\right)^{-1}(u),
$$

where $1_{\omega}$ denotes the characteristic function of the set $\omega$ and $\left(-d^{2} / d x^{2}\right)^{-1}$ the inverse of the operator $-d^{2} / d x^{2}$ in $\omega$ with Dirichlet boundary conditions. This damping mechanism satisfies $\int_{I} B u u d x=\|u\|_{H^{-1}(\omega)}^{2}$ and suffices to guarantee the exponential decay even when $L \in \mathcal{E}$ provided $\omega$ is an open nonempty subset of $I$. 
3. The nonlinear KdV equation with local damping. In this section we analyse the asymptotic behavior of the solutions of the following problem:

$$
\left\{\begin{array}{l}
u_{t}+u_{x}+u_{x x x}+u u_{x}+a(x) u=0 \text { in } I \times(0, \infty), \\
u(0, t)=u(L, t)=0 \text { for all } t>0 \\
u_{x}(L, t)=0 \text { for all } t>0 \\
u(x, 0)=u_{0}(x) \text { for all } x \in I,
\end{array}\right.
$$

where $a=a(x)$ is a nonnegative function belonging to $L^{\infty}(0, L)$ such that $a(x) \geq a_{0}>0$ a.e. in an open, nonempty subset $\omega$ of $I=(0, L)$.

3.1. Existence and uniqueness. Problem (3.1) has a (global) mild solution. More precisely, for any $u_{0} \in L^{2}(I)$ there exists a unique solution $u \in C\left([0,+\infty) ; L^{2}(I)\right) \cap$ $L_{\text {loc }}^{2}\left(0,+\infty ; H_{0}^{1}(I)\right)$. For the sake of completeness we give a sketch of the proof of this result.

We observe that the energy

$$
E(t)=\frac{1}{2} \int_{0}^{L}|u(x, t)|^{2} d x
$$

satisfies the energy dissipation law

$$
\frac{d E}{d t}=-\int_{0}^{L} a(x)|u(x, t)|^{2} d x-\frac{1}{2}\left|u_{x}(0, t)\right|^{2} \leq 0 .
$$

Consequently, it is sufficient to prove local (in time) existence and uniqueness. Global existence will then be an immediate consequence of the a priori estimate

$$
E\left(t_{1}\right) \leq E\left(t_{2}\right) \leq E(0)=\frac{1}{2}\left\|u_{0}\right\|_{L^{2}(I)}^{2} \text { for all } t_{1}>t_{2}>0
$$

that (3.3) provides.

Let us denote by $\{S(t)\}_{t \geq 0}$ the semigroup of contractions that the underlying linear equation satisfies. According to the results of Section 2 we know that $S(\cdot)$ satisfies the following properties:

$$
\begin{gathered}
\left\|S(t) u_{0}\right\|_{L^{2}(I)} \leq\left\|u_{0}\right\|_{L^{2}(I)} \quad \forall t \geq 0, \quad \forall u_{0} \in L^{2}(I) ; \\
\left\|S(\cdot) u_{0}\right\|_{L^{2}\left(0, T ; H_{0}^{1}(I)\right)} \leq C(1+\sqrt{T})\left\|u_{0}\right\|_{L^{2}(I)}, \quad \forall T>0, \quad \forall u_{0} \in L^{2}(I) .
\end{gathered}
$$

Using the variation of constants formula, system (3.1) may be written in the following integral form:

$$
u(t)=S(t) u_{0}+\int_{0}^{t} S(t-s)\left[u u_{x}\right](s) d s=[\phi(u)](t) .
$$

In order to prove local existence and uniqueness, it is sufficient to check that the nonlinear map $\phi$ is a contraction from $X_{T}=L^{\infty}\left(0, T ; L^{2}(I)\right) \cap L^{2}\left(0, T ; H_{0}^{1}(I)\right)$ into itself when $T>0$ is small enough (depending on the size of the initial data $u_{0}$ ).

First of all, we observe that $\phi$ maps continuously $X_{T}$ into itself. Indeed, according to (3.6), $S(\cdot) u_{0}$ lies in $X_{T}$. On the other hand, the function

$$
y(t)=\int_{0}^{t} S(t-s)\left[u u_{x}\right](s) d s
$$


solves the system:

$$
\left\{\begin{array}{l}
y_{t}+y_{x}+y_{x x x}=u u_{x}=f \quad \text { in } I \times(0, T), \\
y(0, t)=y(L, t)=0, \quad t \in(0, T), \\
y_{x}(L, t)=0, \quad t \in(0, T), \\
y(x, 0)=0, \quad x \in I .
\end{array}\right.
$$

Then, by Proposition 4.1, in Rosier [15], it follows that $y$ belongs to $X_{T}$ and the map $f \in L^{1}\left(0, T ; L^{2}(I)\right) \rightarrow y \in X_{T}$ is continuous. Moreover, the map that to each $u \in$ $L^{2}\left(0, T ; H_{0}^{1}(I)\right)$ associates $f=u u_{x} \in L^{1}\left(0, T ; L^{2}(I)\right)$ is continuous as well. This shows that $\phi$ maps continuously $X_{T}$ into itself.

Let us now prove that $\phi$ is a contraction in a suitable ball of $X_{T}$ when $T>0$ is small enough. Obviously,

$$
\phi(u)-\phi(v)=\int_{0}^{t} S(t-s)\left[u u_{x}(s)-v v_{x}(s)\right] d s .
$$

According to the previous analysis,

$$
\|\phi(u)-\phi(v)\|_{X_{T}} \leq 2\left\|u u_{x}-v v_{x}\right\|_{L^{1}\left(0, T ; L^{2}(I)\right)} .
$$

So, applying triangular inequality and Hölder's inequality, we have

$$
\begin{aligned}
\|\phi(u)-\phi(v)\|_{X_{T}} \leq & 2\|u-v\|_{L^{2}\left(0, T ; L^{\infty}(I)\right)}\left\|u_{x}\right\|_{L^{2}\left(0, T ; L^{2}(I)\right)} \\
& +2\|v\|_{L^{2}\left(0, T ; L^{\infty}(I)\right)}\left\|u_{x}-v_{x}\right\|_{L^{2}\left(0, T ; L^{2}(I)\right)} .
\end{aligned}
$$

Let us recall the classical interpolation inequality (Gagliardo-Nirenberg)

$$
\|u\|_{L^{\infty}(I)} \leq C\|u\|_{L^{2}(I)}^{\frac{1}{2}}\left\|u_{x}\right\|_{L^{2}(I)}^{\frac{1}{2}}, \quad \forall u \in H_{0}^{1}(I) .
$$

As a consequence of (3.12) we have

$$
\|u\|_{L^{2}\left(0, T ; L^{\infty}(I)\right)} \leq C T^{\frac{1}{4}}\|u\|_{L^{\infty}\left(0, T ; L^{2}(I)\right)}^{\frac{1}{2}}\|u\|_{L^{2}\left(0, T ; H_{0}^{1}(I)\right)}^{\frac{1}{2}} .
$$

Combining (3.11) and (3.13), we deduce that

$$
\|\phi(u)-\phi(v)\|_{X_{T}} \leq C T^{\frac{1}{4}}\left[\|u\|_{X_{T}}+\|v\|_{X_{T}}\right]\|u-v\|_{X_{T}} .
$$

This shows that $\phi$ is a contraction in the ball $B_{R}$ of $X_{T}$ if

$$
2 C T^{\frac{1}{4}} R<1 \text {. }
$$

Therefore, the proof will be complete if we show that for a suitable choice of $R$ and $T$ satisfying (3.15), the map $\phi$ sends $B_{R}$ into itself. Putting all the previous estimates together, we have

$$
\|\phi(u)\|_{X_{T}} \leq C(1+\sqrt{T})\left\|u_{0}\right\|_{L^{2}(I)}+C T^{\frac{1}{4}}\|u\|_{X_{T}}^{2}
$$

or

$$
\|\phi(u)\|_{X_{T}} \leq C(1+\sqrt{T})\left\|u_{0}\right\|_{L^{2}(I)}+C T^{\frac{1}{4}} R^{2}, \quad \forall u \in B_{R} .
$$

We then take $R=4 C\left\|u_{0}\right\|_{L^{2}(I)}$. For this choice of $R$, the estimate above becomes

$$
\|\phi(u)\|_{X_{T}} \leq C\left[1+\sqrt{T}+16 C^{2} T^{\frac{1}{4}}\left\|u_{0}\right\|_{L^{2}(I)}\right]\left\|u_{0}\right\|_{L^{2}(I)} .
$$


In order to guarantee that the right-hand side of (3.17) is less than $R$, we need to choose $T>0$ sufficiently small such that

$$
\sqrt{T}+16 C^{2} T^{\frac{1}{4}}\left\|u_{0}\right\|_{L^{2}(I)} \leq 3,
$$

which is always possible. Taking $T>0$ possibly smaller allows us to guarantee (3.15) as well.

This concludes the proof of the local existence and uniqueness and, as we mentioned above, the global existence as well due to the a priori bound on the $L^{2}(I)$-norm of the solutions.

In the next subsection we prove our main result on the exponential decay of solutions of $(3.1)$.

3.2. The exponential decay. The aim of this subsection is to show, under certain conditions on the open subset $\omega$ of $I$ (where the damping term is effective), the locally uniform exponential decay of the energy $E(t)$. By that we mean that the exponential decay rate will be uniform for initial data in balls $B_{R}$ of $L^{2}(I)$. But we do not provide any estimate on how the decay rate depends on the radius $R$ of the ball. This has been done, as far as we know, in very few cases and always using some structural condition on the nonlinearity. We refer to [28] for the case of the semilinear wave equation with localized damping in which the uniform exponential decay is proved and to [16] for the analysis of the von Kármán system of thermoelastic plates where an explicit estimate on how the decay rate tends to zero as $R \rightarrow \infty$ is provided.

We employ the method developed in subsection 2.2. However, we may not apply Holmgren's Uniqueness Theorem since we are now dealing with a semilinear equation. In order to conclude we need a unique continuation property (UCP) for the semilinear equation. Let us assume by now that the set $\omega$ where $a>0$ is such that the following holds:

(UCP) If $v \in L^{2}\left(0, T ; H_{0}^{1}(I)\right) \cap L^{\infty}\left(0, T ; L^{2}(I)\right)$ solves

$$
\left\{\begin{array}{l}
v_{t}+v_{x}+v_{x x x}+\lambda v v_{x}=0 \quad \text { in } I \times(0, T), \\
v(0, t)=v(L, t)=0, \quad t \in(0, T), \\
v_{x}(0, t)=v_{x}(L, t)=0, \quad t \in(0, T), \\
v \equiv 0 \quad \text { in } \omega \times(0, T)
\end{array}\right.
$$

with $\lambda \geq 0$ and $T>0$, then necessarily $v \equiv 0$ in $I \times(0, T)$.

Obviously, the only difficulty when checking (UCP) arises when $\lambda>0$. Otherwise the system under consideration is linear with constant coefficients and we can apply Holmgren's Uniqueness Theorem for any open nonempty subset $\omega$ of $I$. The case $\lambda>0$ can be reduced to $\lambda=1$ by scaling. We shall discuss below this (UCP) in detail.

For the moment, assuming that $\omega$ satisfies the (UCP), we have the following:

TheOREm 3.1. Assume that $\omega$ is such that (UCP) holds. Let $a=a(x)$ be a nonnegative function belonging to $L^{\infty}(0, L)$ such that $a(x) \geq a_{0}>0$ a.e. in $\omega$. Then, for any $L>0$ and $R>0$, there exist $c>0$ and $\mu>0$ such that

$$
E(t) \leq c\left\|u_{0}\right\|_{L^{2}(I)}^{2} e^{-\mu t}
$$

for all $t \geq 0$ and any solution of $(3.1)$ with $u_{0} \in L^{2}(I)$ such that $\left\|u_{0}\right\|_{L^{2}(I)} \leq R$. 
REMARK 3.1.

(a) The constants $c$ and $\mu$ appearing in the decay rate depend in particular on the radius $R$ of the ball where the initial data lies. Obtaining estimates on how these constants depend on $R$ is an interesting open problem.

(b) As indicated in Remark 2.2, the damping term $a(\cdot) u$ may be replaced by any other damping mechanism $B u, B$ being a bounded operator in $L^{2}(I)$ such that $B u \equiv 0$ implies that $u=0$ in $\omega$.

There is a simple sufficient condition for (UCP) to hold:

Theorem 3.2. If $\omega$ contains two sets of the form $(0, \delta)$ and $(L-\delta, L)$ for some $\delta>0$, then (UCP) holds.

As an immediate consequence of Theorems 3.1 and 3.2 the following holds:

Corollary 3.1. If $\omega$ contains two sets of the form $(0, \delta)$ and $(L-\delta, L)$ for some $\delta>0$, then the solutions of (3.1) decay locally exponentially uniformly to zero in the sense of Theorem 3.1.

REMARK 3.2. As indicated in the introduction, the main difficulty to check (UCP) is the weak regularity of the solutions under consideration. The existing results on unique continuation (see [21] and [26]) require, to our knowledge, the solution $v$ to be in $L^{\infty}\left(0, T ; H^{s}(I)\right)$ with $s>3 / 2$. Therefore, the main task when checking (UCP) is to show that the weak solution $v$ of (3.18) has, in fact, this regularity property. It is precisely at this point where we use the fact that $\omega$ contains a neighborhood of both extremes $x \equiv 0, L$. We shall return to this issue in Section 4 .

Proof of Theorem 3.1. We proceed as in the proof of Theorem 2.2.

We claim that for any $T>0$ and $R>0$ there exist a positive constant $c=c(R, T)>0$ such that

$$
\left\|u_{0}\right\|_{L^{2}(I)}^{2} \leq c\left[\int_{0}^{T}\left|u_{x}(0, t)\right|^{2} d t+2 \int_{0}^{T} \int_{0}^{L} a(x)|u(x, t)|^{2} d x d t\right],
$$

for every solution of (3.1) with $\left\|u_{0}\right\|_{L^{2}(I)} \leq R$.

This fact, together with the energy dissipation law (3.3) and the semigroup property, suffices to obtain the locally uniform exponential decay.

To prove (3.19) we multiply the equation in problem (3.1) by $x u$ and integrate over $I \times(0, T)$ to obtain

$$
\begin{aligned}
\int_{0}^{T} \int_{0}^{L} u_{x}^{2} d x d t & +\frac{1}{3} \int_{0}^{L} x u^{2}(x, T) d x+\frac{2}{3} \int_{0}^{T} \int_{0}^{L} x a(x) u^{2} d x d t \\
& =\frac{1}{3} \int_{0}^{T} \int_{0}^{L} u^{2} d x d t+\frac{1}{3} \int_{0}^{L} x u_{0}^{2} d x-\frac{2}{3} \int_{0}^{T} \int_{0}^{L} x u^{2} u_{x} d x d t .
\end{aligned}
$$

Integrating by parts and using the boundary conditions we get

$$
\int_{0}^{T} \int_{0}^{L} x u^{2} u_{x} d x d t=-\frac{1}{3} \int_{0}^{T} \int_{0}^{L}|u(x, t)|^{3} d x d t .
$$


So, replacing (3.21) into (3.20) we have the following inequality:

$$
\|u\|_{L^{2}\left(0, T ; H_{0}^{1}(I)\right)}^{2} \leq \frac{(3 T+L)}{3}\left\|u_{0}\right\|_{L^{2}(I)}^{2}+\frac{2}{9} \int_{0}^{T} \int_{0}^{L}|u(x, t)|^{3} d x d t .
$$

Now, by (3.3) and the Sobolev embedding theorem we obtain

$$
\int_{0}^{T} \int_{0}^{L}|u(x, t)|^{3} d x d t \leq C \sqrt{T}\left\|u_{0}\right\|_{L^{2}(I)}^{2}\|u\|_{L^{2}\left(0, T ; H_{0}^{1}(I)\right)},
$$

for some positive constant $C$. Replacing the above inequality in (3.22) we have

$$
\|u\|_{L^{2}\left(0, T ; H_{0}^{1}(I)\right)}^{2} \leq \frac{(8 T+2 L)}{3}\left\|u_{0}\right\|_{L^{2}(I)}^{2}+\frac{(3 T C)}{81}\left\|u_{0}\right\|_{L^{2}(I)}^{4} .
$$

On the other hand, multiplying the equation in (3.1) by $(T-t) u$ and integrating in $I \times(0, T)$, we obtain

$$
\begin{aligned}
T \int_{0}^{L} u_{0}^{2} d x= & \int_{0}^{T} \int_{0}^{L}|u|^{2} d x d t+\int_{0}^{T}(T-t)\left|u_{x}(0, t)\right|^{2} d t \\
& +2 \int_{0}^{T} \int_{0}^{L}(T-t) a(x)|u|^{2} d x d t
\end{aligned}
$$

because $\int_{0}^{T} \int_{0}^{L}(T-t) u^{2} u_{x} d x d t=0$. From (3.24) we deduce that

$$
\begin{aligned}
\left\|u_{0}\right\|_{L^{2}(I)}^{2} \leq & \frac{1}{T} \int_{0}^{T} \int_{0}^{L}|u|^{2} d x d t+\int_{0}^{T}\left|u_{x}(0, t)\right|^{2} d t \\
& +2 \int_{0}^{T} \int_{0}^{L} a(x)|u|^{2} d x d t .
\end{aligned}
$$

Thus, in order to prove (3.19) it is sufficient to show that for any $T>0$ and $R>0$, there exists a positive constant $C_{1}=C_{1}(R, T)$ such that

$$
\int_{0}^{T} \int_{0}^{L}|u|^{2} d x d t \leq C_{1}\left\{\int_{0}^{T}\left|u_{x}(0, t)\right|^{2} d t+2 \int_{0}^{T} \int_{0}^{L} a(x)|u|^{2} d x d t\right\}
$$

for any solution of (3.1) with $\left\|u_{0}\right\|_{L^{2}(I)} \leq R$.

We argue by contradiction. Suppose that (3.26) does not hold. Then, there exists a sequence of functions $u_{n} \in L^{\infty}\left(0, T ; L^{2}(I)\right) \cap L^{2}\left(0, T ; H_{0}^{1}(I)\right)$ that solve (3.1), satisfying $\left\|u_{n}(\cdot, 0)\right\|_{L^{2}(I)}^{2} \leq R$ and such that

$$
\lim _{n \rightarrow+\infty} \frac{\left\|u_{n}\right\|_{L^{2}\left(0, T ; L^{2}(I)\right)}^{2}}{\int_{0}^{T}\left|\frac{\partial}{\partial x} u_{n}(0, t)\right|^{2} d t+2 \int_{0}^{T} \int_{0}^{L} a(x)\left|u_{n}\right|^{2} d x d t}=+\infty .
$$

Let $\lambda_{n}=\left\|u_{n}\right\|_{L^{2}\left(0, T: L^{2}(I)\right)}$ and define $v_{n}(x, t)=u_{n}(x, t) / \lambda_{n}$. For each $n \in \mathbb{N}$ the function $v_{n}$ satisfies

$$
\begin{aligned}
& \left\{\begin{array}{l}
\left(v_{n}\right)_{t}+\left(v_{n}\right)_{x}+\left(v_{n}\right)_{x x x}+\lambda_{n}\left(v_{n}\right)\left(v_{n}\right)_{x}+a(x) v_{n}=0 \quad \text { in } I \times(0, T) \\
v_{n}(0, t)=v_{n}(L, t)=0 \\
\left(v_{n}\right)_{x}(L, t)=0,
\end{array} \quad\left\|v_{n}\right\|_{L^{2}\left(0, T: L^{2}(I)\right)}=1\right.
\end{aligned}
$$


and

$$
\int_{0}^{T}\left|\frac{\partial}{\partial x} v_{n}(0, t)\right|^{2} d t+2 \int_{0}^{T} \int_{0}^{L} a(x)\left|v_{n}\right|^{2} d x d t \rightarrow 0
$$

as $n \rightarrow+\infty$.

Using (3.25) it follows that $v_{n}(\cdot, 0)$ is bounded in $L^{2}(I)$. By $(3.23)$ it follows that

$$
\left\|v_{n}\right\|_{L^{2}\left(0, T ; H_{0}^{1}(I)\right)} \leq C, \quad \forall n \in \mathbb{N}
$$

for some constant $C>0$. On the other hand, $v_{n}\left(v_{n}\right)_{x}$ belongs to $L^{2}\left(0, T ; L^{1}(I)\right)$ and moreover

$$
\left\|v_{n}\left(v_{n}\right)_{x}\right\|_{L^{2}\left(0, T ; L^{1}(I)\right)} \leq\left\|v_{n}\right\|_{L^{\infty}\left(0, T ; L^{2}(I)\right)}\left\|v_{n}\right\|_{L^{2}\left(0, T ; H_{0}^{1}(I)\right)} .
$$

So, by (3.31) we obtain that there exists $C>0$ such that

$$
\left\|v_{n}\left(v_{n}\right)_{x}\right\|_{L^{2}\left(0, T ; L^{1}(I)\right)} \leq C .
$$

Since $\left\{\lambda_{n}\right\}$ is a bounded sequence (because $\left.\left\|u_{n}(\cdot, 0)\right\|_{L^{2}(I)} \leq R\right)$, it follows by (3.28), (3.29), (3.31), and (3.32) that $\left\{\left(v_{n}\right)_{t}\right\}$ is bounded in $L^{2}\left(0, T ; H^{-2}(I)\right)$.

Then, using (3.31) and classical compactness results (see [22]) we can extract a subsequence of $\left\{v_{n}\right\}$, which we also denote by $\left\{v_{n}\right\}$, such that

$$
v_{n} \rightarrow v \quad \text { strongly in } L^{2}\left(0, T ; L^{2}(I)\right)
$$

and by $(3.29)$

$$
\|v\|_{L^{2}\left(0, T ; L^{2}(I)\right)}=1
$$

Moreover

$$
\begin{aligned}
0 & =\liminf _{k \rightarrow \infty}\left\{\int_{0}^{T}\left|\frac{\partial}{\partial x} v_{n}(0, t)\right|^{2} d t+2 \int_{0}^{T} \int_{0}^{L} a(x)\left|v_{n}\right|^{2} d x d t\right\} \\
& \geq \int_{0}^{T}\left|\frac{\partial}{\partial x} v(0, t)\right|^{2} d t+2 \int_{0}^{T} \int_{0}^{L} a(x)|v|^{2} d x d t
\end{aligned}
$$

and we may deduce that $a(x) v \equiv 0$ in $I \times(0, T)$. So $v \equiv 0$ in $\omega \times(0, T)$ and, furthermore, $v_{x}(0, t)=0$ in $(0, T)$.

We now distinguish the following two situations:

a) There exists a subsequence of $\left\{\lambda_{n}\right\}$ also denoted by $\left\{\lambda_{n}\right\}$ such that

$$
\lambda_{n} \rightarrow 0 \text {. }
$$

In this case, the limit $v$ satisfies the linear problem

$$
\left\{\begin{array}{l}
v_{t}+v_{x}+v_{x x x}=0 \quad \text { in } I \times(0, T) \\
v(0, t)=v(L, t)=0 \quad \text { for all } t \in(0, T), \\
v_{x}(0, t)=v_{x}(L, t)=0 \quad \text { for all } t \in(0, T), \\
v(x, t)=0 \quad(x, t) \text { in } \omega \times(0, T)
\end{array}\right.
$$

Then, by Holmgren's Uniqueness Theorem, $v \equiv 0$ in $I \times(0, T)$ and this contradicts (3.33).

b) There exists a subsequence of $\left\{\lambda_{n}\right\}$ also denoted by $\left\{\lambda_{n}\right\}$ and $\lambda>0$ such that

$$
\lambda_{n} \rightarrow \lambda \text {. }
$$


In this case, the limit function $v$ solves system (3.28) and so, by the (UCP) assumed to hold for the subset $\omega$, we have $v \equiv 0$ in $I \times(0, T)$ and again, this is a contradiction.

Recapitulating, we see that, in each of these possible situations (a) and (b) we are in a contradiction. Then, necessarily, (3.26) holds. This completes the proof of Theorem 3.1 .

REMARK 3.3. Note that we have used in an essential way the fact that the initial data are a priori restricted to a ball $B_{R}$. Otherwise, in the contradiction argument, we would have also to address the case where $\lambda_{n} \rightarrow \infty$. We were not able to do this. As far as we know, this has only been done in [28] in the context of the semilinear wave equation with localized damping under suitable structural assumptions on the nonlinearity.

Proof of Theorem 3.2. Since $v \in L^{2}\left(0, T ; H_{0}^{1}(I)\right) \cap L^{\infty}\left(0, T ; L^{2}(I)\right)$, we obtain $v_{t} \in$ $L^{2}\left(0, T ; H^{-2}(I)\right)$. So, by Lions-Magenes [11] (vol. 1) and Teman [25] (ch. III, lemma 1.4) we know that $v$ is weakly continuous from $[0, T]$ into $L^{2}(I)$.

Recall that, according to the structure of $\omega, v \equiv 0$ in $\{(0, \delta) \cup(L-\delta, L)\} \times(0, T)$. Now, let us introduce the extended function

$$
V(x, t)= \begin{cases}v(x, t) & \text { if }(x, t) \in(\delta, L-\delta) \times(0, T), \\ 0 & \text { if }(x, t) \in\{\mathbb{R}-(\delta, L-\delta)\} \times(0, T) .\end{cases}
$$

Then $V$ satisfies

$$
\begin{cases}V_{t}+V_{x}+V_{x x x}+\lambda V V_{x}=0 & \text { in } \mathbb{R} \times(0, T), \\ V(x, 0)=\phi(x) & \text { in } \mathbb{R}\end{cases}
$$

where

$$
\phi(x)= \begin{cases}v(x, 0) & \text { if } x \in(\delta, L-\delta) \\ 0 & \text { if } x \in\{\mathbb{R}-(\delta, L-\delta)\}\end{cases}
$$

If we consider $W(x, t)=V(x+t, t)$, then $W$ solves

$$
\begin{cases}W_{t}+W_{x x x}+\lambda W W_{x}=0 & \text { in } \mathbb{R} \times(0, T), \\ W(x, 0)=\phi(x) & \text { in } \mathbb{R} .\end{cases}
$$

Since $\phi$ has compact support and belongs to $L^{2}(\mathbb{R})$,

$$
\int_{\mathbb{R}} \phi^{2}(x) \exp (2 b x) d x<\infty \quad \forall b>0 .
$$

Thus, by the regularizing properties proved by Kato [6] (Thm. 12.1), $W \in C^{\infty}(I \times(0, T))$. Therefore, $V$ is smooth as well and applying the unique continuation result in [26] (Thm. 4.3) we have $V \equiv 0$ and, consequently,

$$
v(x, t) \equiv 0, \quad x \in I, t \in[0, T] .
$$

The proof is complete. 
3.3. Exponential decay of small amplitude solutions. We observe that, whenever the underlying semigroup decays exponentially, small amplitude solutions decay exponentially as well. This fact can easily be proved as follows.

Let $S(\cdot)$ be the linear semigroup associated with (3.1). We know that

$$
\|S(t)\|_{\mathcal{L}\left(L^{2}(I), L^{2}(I)\right)} \leq C e^{-\mu t}
$$

in two cases:

(a) with $a \equiv 0$ when $L \notin \mathcal{E}$;

(b) with $a \geq a_{0}>0$ a.e. in an open nonempty subset $\omega$ of $(0, L)$ when $L \in \mathcal{E}$. Let $T>0$ and $\gamma<1$ be such that

$$
\|S(T)\|_{\mathcal{L}\left(L^{2}(I), L^{2}(I)\right)}=\gamma<1 .
$$

Solutions of the nonlinear problem satisfy the variation of constants formula (3.7). In particular, for $t=T$ we have

$$
u(T)=S(T) u_{0}+\int_{0}^{T} S(T-s)\left[u u_{x}\right](s) d s .
$$

Moreover, it follows by (3.35) that

$$
\|u(T)\|_{L^{2}(I)} \leq \gamma\left\|u_{0}\right\|_{L^{2}(I)}+\int_{0}^{T}\left\|u u_{x}(s)\right\|_{L^{2}(I)} d s
$$

and so

$$
\|u(T)\|_{L^{2}(I)} \leq \gamma\left\|u_{0}\right\|_{L^{2}(I)}+\frac{1}{2}\|u\|_{L^{2}\left(0, T ; H_{0}^{1}(I)\right)}^{2} .
$$

However, in view of (3.23) we also have

$$
\|u\|_{L^{2}\left(0, T ; H_{0}^{1}(I)\right)}^{2} \leq C\left[\left\|u_{0}\right\|_{L^{2}(I)}^{2}+\left\|u_{0}\right\|_{L^{2}(I)}^{4}\right] .
$$

Combining the last two estimates, we obtain

$$
u(T)_{L^{2}(I)} \leq\left\|u_{0}\right\|_{L^{2}(I)}\left[\gamma+C\left[\left\|u_{0}\right\|_{L^{2}(I)}+\left\|u_{0}\right\|_{L^{2}(I)}^{3}\right]\right] .
$$

We see that, taking

$$
C\left[\left\|u_{0}\right\|_{L^{2}(I)}+\left\|u_{0}\right\|_{L^{2}(I)}^{3}\right]<\frac{1-\gamma}{2},
$$

the following holds:

$$
\|u(T)\|_{L^{2}(I)} \leq \frac{1+\gamma}{2}\left\|u_{0}\right\|_{L^{2}(I)} .
$$

This fact together with the semigroup property implies the exponential decay of solutions with small enough initial data satisfying (3.38).

Obviously this argument provides an exponential decay, as close as we wish to the one of the linear semigroup, when the size of the initial data tends to zero.

This argument fails for large solutions, whose analysis has been performed in subsection 3.2 by a different method. 
4. Some open problems. The results in Section 3 leave open the following interesting problem. What are the minimal assumptions on the open nonempty subset of $\omega$ of $I$ for (UCP) to hold?

As we have seen in the proof of Theorem 3.2, the main difficulty to achieve (UCP) is to improve the regularity of $v$. When $\omega$ contains a neighborhood of both extremes $x=0, L$ we have been able to do this since, extending the solution by zero outside of $I$, we have built a solution of the Cauchy problem for the KdV equation on the whole line and therefore we have been able to apply the existing classical regularization properties. However, when $\omega$ does not have this property and, in particular, when $\omega$ contains only a neighborhood of one of the extremes or lies in the interior of $I$, this argument may not be applied. Then one has to improve the regularity of the solution working in the bounded interval $(0, L)$. This remains to be done. Of course, one could also try to prove directly a unique continuation result for weak solutions using Carleman inequalities. But this remains to be done as well.

On the other hand, in view of the energy dissipation law (3.3), one may also consider the problem of whether solutions of (3.1) tend to zero as $t \rightarrow+\infty$ when $a \equiv 0$. Applying the arguments in Section 3, the problem would reduce to seeing if the unique solution of

$$
\left\{\begin{array}{l}
v_{t}+v_{x}+v_{x x x}+\lambda v v_{x}=0 \quad \text { in } I \times(0, T), \\
v(0, t)=v(L, t)=0 \quad t \in(0, T), \\
v_{x}(L, t)=0 \quad t \in(0, T)
\end{array}\right.
$$

satisfying the extra condition

$$
v_{x}(0, t)=0 \text { for } t \in(0, T)
$$

with $\lambda \geq 0$, is the trivial one.

As shown in Section 2, when $\lambda=0$ and $L \in \mathcal{E}$ there are nontrivial solutions of (4.1) (4.2).

When $L$ does not belong to $\mathcal{E}$, we know that nontrivial solutions of (4.1)-(4.2) do not exist when $\lambda=0$. But the problem is open when $\lambda>0$.

Acknowledgments. The first author was partially supported by a Grant of CNPq and PRONEX (MCT, Brasil). The third author was supported by Grants PB 96-0663 of the DGES (Spain) and ERB FMRX CT 960033 of the European Union and partially supported by PRONEX (MCT, Brasil) and FAPERJ (Brasil).

\section{REFERENCES}

[1] J. Bona and R. Winter, The Korteweg-de Vries equation posed in a quarter-plane, SIAM J. Math. Anal. 14, 2056-1106 (1983)

[2] Th. Cazenave and A. Haraux, Introduction aux problèmes d'évolution semi-linéaires, Mathématiques et Applications, Soc. Math. Appl. et Ind., Ellipses, France, 1990

[3] C. M. Dafermos, On the existence and the asymptotic stability of solutions to the equations of linear thermoelasticity, Arch. Rational Mech. Anal. 29, 241-271 (1968)

[4] A. Haraux, Stabilization of trajectories for some weakly damped hyperbolic equations, J. Differential Equations 59, 145-154 (1985)

[5] A. E. Ingham, Some trigonometrical inequalities with application to the theory of series, Math. Z. 31, 367-379 (1936) 
[6] T. Kato, On the Cauchy problem for the (generalized) Korteweg-de Vries equation, Stud. Appl. Math., Adv. in Math. Suppl. Stud. 8, 93-128 (1983)

[7] V. Komornik, Exact controllability and stabilization, the multiplier method, Research in Applied Mathematics 36, John Wiley and Sons, Masson, 1994

[8] V. Komornik, D. L. Russell, and B. Y. Zhang, Stabilisation de l'équation de Korteweg-de Vries, C. R. Acad. Sci. Paris, Série I Math. 312, 841-843 (1991)

[9] S. N. Kruzhkov and A. V. Faminskii, Generalized solutions of the Cauchy problem for the Kortewegde Vries equation, Math. URSS Sbornik 38, 391-421 (1984)

[10] J. L. Lions, Contrôlabilité exacte, perturbations et stabilisation de systèmes distribués, Tome 1, Contrôlabilité exacte, Collection de Recherches en Mathématiques Appliquées, 8, Masson, Paris, 1988

[11] J. L. Lions and E. Magenes, Problèmes aux limites non homogènes et applications, Tome 1, Dunod, Paris, 1968

[12] S. Micu and E. Zuazua, Boundary controllability of a linear hybrid system arising in the control of noise, SIAM J. Control Optim. 35, 1614-1638 (1997)

[13] R. M. Miura, The Korteweg-de Vries equation, A survey of results, SIAM Review 18, 412-459 (1976)

[14] M. Nakao, Decay of solutions of the wave equation with a local nonlinear dissipation, Math. Ann. 305, 403-417 (1996)

[15] L. Rosier, Exact boundary controllability for the Korteweg-de Vries equation on a bounded domain, ESAIM, Control Optimization and Calculus of Variations, vol. 2, 1997, pp. 33-55 (electronic)

[16] G. Perla-Menzala and E. Zuazua, Decay rates for the von Kármán system of thermoelastic plates, Integral and Differential Equations 11, 755-770 (1998) (C. R. Acad. Sci. Paris 324, $49-54$ (1997))

[17] A. Ruiz, Unique continuation for weak solutions of the wave equation plus a potential, J. Math. Pures Appl. 71, 455-467 (1992)

[18] D. L. Russell and B. Y. Zhang, Smoothing and decay properties of solutions of the Korteweg-de Vries equation on a periodic domain with point dissipation, J. Math. Anal. Appl. 190, 449-488 (1995)

[19] D. L. Russell and B. Y. Zhang, Controllability and stabilizability of the third order linear dispersion equation on a periodic domain, SIAM J. Control. Optim. 31, 659-676 (1993)

[20] D. L. Russell and B. Y. Zhang, Exact controllability and stabilization of the Korteweg-de Vries equation, Trans. Amer. Math. Soc. 348, 3643-3672 (1996)

[21] J. C. Saut and B. Scheurer, Unique continuation for some evolution equations, J. Differential Equations 66, 118-139 (1987)

[22] J. Simon, Compact sets in the space $L^{p}(0, T ; B)$, Annali di Matematica Pura ed Applicata (IV), vol. CXLVI, 1987, pp. 65-96

[23] M. Slemrod, Weak asymptotic decay via a "Relaxed Invariance Principle" for a wave equation with nonlinear, nonmonotone damping, Proc. Royal Soc. Edinburgh 113, 87-97 (1989)

[24] R. Temam, Sur un problème non linéaire, J. Math. Pures et Appl. 38, 157-172 (1969)

[25] R. Teman, Navier-Stokes Equations, Studies in Mathematics and its Applications 2, North-Holland, 1977

[26] B. Y. Zhang, Unique continuation for the Korteweg-de Vries equation, SIAM J. Math. Anal. 23, 55-71 (1991)

[27] B. Y. Zhang, Exact boundary controllability of the Korteweg-de Vries equation, SIAM J. Control Optim. 37, 548-565 (1999)

[28] E. Zuazua, Exponential decay for the semilinear wave equation with locally distributed damping, Comm. Partial Differential Equations 15 (2), 205-235 (1990)

[29] E. Zuazua, Contrôlabilité exacte en un temps arbitrairement petit de quelques modèles de plaques, Appendix 1 in [10], Tome I, pp. 465-491 